\title{
Logistic map driven by dichotomous noise
}

\author{
J. M. Gutierrez and A. Iglesias \\ Departamento de Matemática Aplicada y Ciencias de la Computación, Universidad de Cantabria, \\ Avenida de los Castros, s/n, 39005 Santander, Spain \\ M. A. Rodriguez \\ Departamento de Física Moderna, Universidad de Cantabria, Avenida de los Castros, s/n, 39005 Santander, Spain
}

(Received 4 January 1993; revised manuscript received 10 May 1993)

\begin{abstract}
Bifurcation diagrams and invariant densities are computed and interpreted for a logistic map driven by dichotomous noise. Two deterministic limits are analyzed. Changes in the stability of such a system, when varying the correlation time of the noise, are numerically studied. The peaks of the invariant density in the white-noise case are identified as originating from the most stable attractors among those appearing in the deterministic limits.
\end{abstract}

PACS number(s): 05.40. $+\mathrm{j}, 05.45 .+\mathrm{b}, 02.50 .-\mathrm{r}$

\section{INTRODUCTION}

The influence of noise on discrete dynamical systems has been investigated from several points of view. There are several investigations where the shift, broadening, and suppression of bifurcations have been analyzed [1]. Others deal with the scaling of Lyapunov exponents and invariant densities near the onset of chaos [2,3]. Most of these effects have been investigated with a Gaussian white noise with small intensity. The noise acts as a perturbation of the original system and obviously their effects must be related with changes on this system.

In this paper, we consider a different kind of noise acting on discrete systems. Instead of a Gaussian white noise, we deal with multilevel processes with arbitrary intensity and correlation time.

In general, a map driven by discrete dichotomous noise comes from continuous systems, driven by a random sequence of pulses with constant width. Despite its importance in fields related to electronics, this kind of stochastic signal has not been used in the context of stochastic processes. Explicitly, for a one-dimensional continuous system given by the equation

$$
\frac{d x}{d t}=f(x, \rho(t))
$$

$\rho(t)$ being a noise composed by random squared pulses, with amplitude $+\Delta$ or $-\Delta$ and width $z_{0}$, the values of $x$ at the beginning of the pulse $\left\{x(0), x\left(z_{0}\right), \ldots, x\left(n z_{0}\right)\right\}$ follow a stochastic map:

$$
x\left(n z_{0}\right)=Y\left(x\left((n-1) z_{0}\right), z_{0}, \rho_{n-1}\right)
$$

with $\rho_{n}= \pm \Delta$ and $Y\left(x_{0}, t, \rho_{n}\right)$ being the solution of (1) with $x_{0}$ as initial condition at $t=0$. In the case of a regular system with a stable attractor point and with a value of $z_{0}$ close to the characteristic time of the system, the map becomes of the logistic kind.

A colored noise with sufficient intensity can induce dramatic effects on the original attractors, changing their character or creating different ones. These changes can be produced by randomness and by memory effects. Changes due only to memory effects on a logistic map have been reported in a recent paper [4]. In fact, the bifurcation scenario found in the aforementioned paper is rather similar to our scenario for some values of the correlation time. Changes due to colored noise have been extensively studied in continuous systems with Gaussian or Markovian noise but only a few works are devoted to maps. It is worth remarking on the recent work of Ref. [5], analyzing the noise-induced transitions in a class of bistable oscillators driven by dichotomous noise. As pointed out in this paper, a dichotomic noise is a good candidate for studying such a complicated problem. As is well known from the study of one-dimensional stochastic continuous models [6], the simplicity of this noise allows one to derive analytical expressions for the probability density or transition rates for some nonlinear models. We show that also, in discrete systems with dichotomic noise, one is able to derive analytical results.

In Sec. II we introduced the model: a logistic map driven by a discrete dichotomic noise. Reasons for this choice are rather obvious: of all the models in dynamical systems, none is as simple or as widely studied as the logistic map. Moreover, it is quite amenable to both theoretical and numerical techniques and, at the same time, it exhibits all the nonlinear behavior of more complicated systems. On the other hand, the dichotomic noise is also a widely used noise for driving systems. It is feasible to produce, simple to simulate, and accessible to analyze.

Following with the description of the system we remark that the noise drives the system in a multiplicative manner. Two parameters $\pm \Delta$, the value of the noise, and $\alpha$, the conditional probability of repeating the same value of the noise, completely define the noise process. When varying $\alpha$ from 0 to 1 several interesting situations appear. The values $\alpha \rightarrow 0$ and $\alpha \rightarrow 1$ correspond to two deterministic limits. In the case $\alpha=\frac{1}{2}$, we have a white noise where all sequences of the noise have the same 
probability. A previous analysis of these situations can be found in Ref. [7].

Section III is devoted to the analysis of bifurcations in presence of dichotomic noise.

In Sec. IV, the stability of the system as a function of the correlation time of the noise is computed in several interesting situations. For a measure of the stability we use Lyapunov exponents as suggested by several authors [8]. We show that in contrast with the one-dimensional continuous model, the white-noise case does not represent the most unstable situation. All transitions, from chaos to regular motion or its inverse, are possible when varying the correlation time. Hence, we show that the effect of a change in the correlation time can be dramatic.

In Sec. V, the white-noise case is considered. We focus on the search for reminiscent situations coming from the deterministic limits. Since most probable realizations are those appearing in the deterministic limits, it seems a good idea to compare the behavior of a given $\alpha$ with that of the deterministic limits $(\alpha \rightarrow 1)$. In this matter, peaks of the invariant density corresponding to effects induced by noise are identified.

To conclude this introduction, we remark on an important point presented in this paper. We believe that the study of noise effects on dynamic systems by means of multilevel processes may be enlightening in order to understand some aspects of the evolution of chaotic systems with noise. In fact, it is possible to describe these effects by superposition of well-defined maps. Clearly, this picture is quite precise.

\section{THE MODEL}

We consider a logistic map with a multiplicative noise in the form

$$
x_{n+1}=\mu\left(1+\xi_{n}\right) x_{n}\left(1-x_{n}\right),
$$

where $\xi$ is a dichotomic noise taking two possible values $\pm \Delta$ with a probability $\alpha \leq 1$ of repeating the same value in the following iteration. Since the noise is bounded, the equation is well defined for $\mu(1+\Delta)<4$.

The parameters $\Delta$ and $\alpha$ completely define the process. $\Delta$ gives the intensity of the noise and $\alpha$ is related to the "correlation time." In a continuous model, the dichotomic noise usually employed is Markovian, which means that the distribution of times where the value of the intensity changes from $\pm \Delta$ to $\mp \Delta$ is exponential. In this case, the correlation time is given by the mean value of these times.

Following on from this, we take as a measure of the correlation time $\tau$, in the discrete case, the average duration of the most probable sequence of the noise. For $\alpha>\frac{1}{2}$, this sequence is formed by repetitive values $+\Delta$ and $-\Delta$, with $\tau$ given by $\alpha /(1-\alpha)$. In this case, the definition of correlation time coincides with the one given in the continuous process. For $\alpha=\frac{1}{2}$ we have a white noise and all sequences are equally weighted. For $\alpha<\frac{1}{2}$ the most probable sequence is formed by alternated values of $+\Delta$ and $-\Delta$. Now $\tau$ reads $(1-\alpha) / \alpha$. This situation does not appear in a continuous model. Hence, in the discrete process there are two deterministic limits, for $\alpha \rightarrow 1$ and $\alpha \rightarrow 0$. Then, the white-noise case, $\alpha=\frac{1}{2}$, can be obtained from one of the above-mentioned limits.

In order to analyze the evolution of the noisy map we start defining the joint probability, $P_{n}(x, \pm \Delta)$ of finding the map and noise with values $x$ and $\pm \Delta$, after $n$ time steps. This joint probability follows a master equation given by

$$
\begin{aligned}
P_{n+1}(x, \pm \Delta)= & \alpha \int \delta\left(x-f_{ \pm}(y)\right) P_{n}(y, \pm \Delta) d y \\
& +(1-\alpha) \int \delta\left(x-f_{\mp}(y)\right) P_{n}(y, \mp \Delta) d y,
\end{aligned}
$$

with $f_{ \pm}(y)=\mu(1 \pm \Delta) y(1-y)$. In the limit $n \rightarrow \infty$ the equation for the stationary joint density $p(x, \pm \Delta)$ is

$$
\begin{aligned}
P(x, \pm \Delta)= & \alpha \int \delta\left(x-f_{ \pm}(y)\right) P(y, \pm \Delta) d y \\
& +(1-\alpha) \int \delta\left(x-f_{\mp}(y)\right) P(y, \mp \Delta) d y .
\end{aligned}
$$

The stationary probability density for the noisy map $\rho(x)$ is obviously given by

$$
\rho(x)=P(x,+\Delta)+P(x,-\Delta) .
$$

With these equations in mind, we now consider the two limiting cases. For $\alpha \rightarrow 1$ we take $\alpha=1-\epsilon$, where $\epsilon$ is a small quantity, and make perturbations in the form

$$
P(x, \pm \Delta)=P_{0}(x, \pm \Delta)+\epsilon P_{1}(x, \pm \Delta) .
$$

Substituting (7) into (5) we have for the zero order in $\epsilon$

$$
P_{0}(x, \pm \Delta)=\int \delta\left(x-f_{ \pm}(y)\right) P_{0}(y, \pm \Delta) d y
$$

and for the first order

$$
\begin{aligned}
P_{1}(x, \pm \Delta)= & \int \delta\left(x-f_{ \pm}(y)\right)\left[P_{0}(y, \pm \Delta)+P_{0}(y, \mp \Delta)\right] d y \\
& +\int \delta\left(x-f_{ \pm}(y)\right) P_{1}(y, \pm \Delta) d y
\end{aligned}
$$

Equation (8) has a normalized solution given by

$$
P_{0}(x, \pm \Delta)=\frac{1}{2} \rho_{ \pm}(x)
$$

where $\rho_{ \pm}(x)$ is the invariant density of the logistic map $f_{ \pm}(x)$, respectively. The integral equation (9) can be iteratively solved. From (6), (10), and an iterative $n$ solution of (9), we finally obtain

$$
\begin{aligned}
\rho(x)= & \frac{1}{2}(1-n \epsilon) \rho_{+}(x)+\frac{1}{2}(1-n \epsilon) \rho_{-}(x) \\
& +\frac{\epsilon}{2}\left[\int \delta\left(x-f_{+}\left(y_{1}\right)\right) \rho_{-}\left(y_{1}\right) d y_{1}+\cdots+\int \cdots \int \delta\left(x-f_{+}\left(y_{1}\right)\right) \cdots \delta\left(y_{n-1}-f_{+}\left(y_{n}\right)\right) \rho_{-}\left(y_{n}\right) d y_{1} \cdots d y_{n}\right] \\
& +\frac{\epsilon}{2}\left[\int \delta\left(x-f_{-}\left(y_{1}\right)\right) \rho_{+}\left(y_{1}\right) d y_{1}+\cdots+\int \cdots \int \delta\left(x-f_{-}\left(y_{1}\right)\right) \cdots \delta\left(y_{n-1}-f_{-}\left(y_{n}\right)\right) \rho_{+}\left(y_{n}\right) d y_{1} \cdots d y_{n}\right] \\
& +O\left(\epsilon^{2}\right) .
\end{aligned}
$$


This solution gives the superposition of $\rho_{+}$and $\rho_{-}$and, in order $\epsilon$, the transient to an attractor of $f_{ \pm}$when starting from an attractor of $f_{\mp}$, respectively.

Let us now consider the limit $\alpha \rightarrow 0$. Taking $\alpha=\epsilon$ in (5) and making an identical perturbative analysis as in the case with $\alpha \rightarrow 1$ we obtain for the zero-order equation

$P_{0}(x, \pm \Delta)=\int \delta\left(x-f_{ \pm}\left(f_{\mp}(y)\right)\right) P_{0}(y, \pm \Delta) d y$

and for the first order

$$
\begin{aligned}
P_{1}(x \pm \Delta)= & \int \delta\left(x-f_{ \pm}(y)\right)\left[P_{0}(y, \pm \Delta)-P_{0}(y, \mp \Delta)\right] d y \\
& +\int \delta\left(x-f_{ \pm}(y)\right) P_{1}(y, \mp \Delta) d y
\end{aligned}
$$

Equation (12) corresponds to the equation for the invariant density of the composed map $f_{+} f_{-}$( or $f_{-} f_{+}$). Since this map is quartic, there would be bistable situation and consequently one or two invariant densities may appear depending on the initial conditions. Hence, a general solution of $(12)$ is

$$
P_{0}(x, \pm \Delta)=\frac{1}{2} \hat{\rho}_{ \pm}(x)=\frac{1}{2} \sum_{i=1}^{I} \frac{1}{I} \rho_{ \pm}^{i}(x),
$$

where $\rho_{ \pm}^{i}(x)$ are the invariant densities in the $(I=1)$ monostable or $(I=2)$ bistable case, respectively. Now Eq. (13) can be solved by iteration. We obtain for the invariant density

$$
\begin{aligned}
& \rho(x)=\frac{1}{2}(1-n \epsilon) \hat{\rho}_{+}(x)+\frac{1}{2}(1-n \epsilon) \hat{\rho}_{-}(x) \\
& +\frac{\epsilon}{2}\left[\int \delta\left(x-f_{+}\left(y_{1}\right)\right) \hat{\rho}_{+}\left(y_{1}\right) d y_{1}+\cdots+\int \cdots \int \delta\left(x-f_{+}\left(y_{1}\right)\right)\right. \\
& \left.\quad \times \delta\left(y_{1}-f_{+}\left(y_{2}\right)\right) \cdots \delta\left(y_{n-1}-f_{+}\left(y_{n}\right)\right) \hat{\rho}_{+}\left(y_{n}\right) d y_{1} \cdots d y_{n}\right] \\
& +\frac{\epsilon}{2}\left[\int \delta\left(x-f_{-}\left(y_{1}\right)\right) \hat{\rho}_{-}\left(y_{1}\right) d y_{1}+\cdots \int \cdots \int \delta\left(x-f_{-}\left(y_{1}\right)\right) \delta\left(y_{1}-f_{-}\left(y_{2}\right)\right) \cdots \delta\left(y_{n-1}-f_{-}\left(y_{n}\right)\right)\right. \\
& \left.\quad \times \hat{\rho}_{-}\left(y_{n}\right) d y_{1} \cdots d y_{n}\right]
\end{aligned}
$$

Summarizing, the limit $\alpha \rightarrow 1$ yields to a superposition of two logistic maps $f_{+}$and $f_{-}$. As a consequence, the invariant measure and the Lyapunov exponent are given by the arithmetic mean: $\rho(x)=\frac{1}{2} \rho_{+}(x)+\frac{1}{2} \rho_{-}(x)$ and $\lambda=\frac{1}{2} \lambda_{+}+\frac{1}{2} \lambda_{-}$, respectively. In Fig. 1(a) we show the bifurcation diagram for $\alpha=0.99$. In agreement with our analysis, it is mainly composed by the superposition of the bifurcation diagrams $f_{-}, f_{+}$and in a lower proportion (first-order contribution) by the transient to an attractor of $f_{ \pm}$when starting from an attractor of $f_{\mp}$.

In the limit $\alpha \rightarrow 0$ we find, in the zero order, the superposition of attractors of the composed maps $f_{-} f_{+}$and $f_{+} f_{-}$. Hence, when these maps are monostable there are two superposed attractors, whereas there will be four in bistable situations. In Fig. 1(c) we have plotted the bifurcation diagram for $\alpha=0.01$. As the analysis predicts, it is formed by the above-mentioned attractors and in a lower proportion (first order) by the transient states to the attractors of $f \pm$ when starting from attractors of $f \mp$, respectively. When $\alpha$ goes to $\frac{1}{2}$ (white-noise limit) transient states from the attractors overlap. In Fig. 1(b) we have plotted the white-noise case. A better description of this case with invariant densities will be given in Sec. IV.

\section{BIFURCATIONS AND NOISE}

The concept of bifurcation in presence of noise is not clear in many situations. Except when the noise is weak and the deterministic framework still remains, it is difficult to determine when a bifurcation takes place. Perhaps the most widely used indicator of the occurrence of a bifurcation would be the appearance of relevant changes in the invariant density. Using this rule, bifurcation of several kinds can be observed in our system either as produced from the deterministic behavior or as induced by the noise. As expected, near the $\alpha=1$ limit there appears a superposition of bifurcations corresponding to the logistic maps $f_{+}$and $f_{-}$. Since the Lyapunov exponent $\lambda$ is the mean of the exponents corresponding to $f_{+}$and $f_{-}\left[\lambda=\left(\lambda_{+}+\lambda_{-}\right) / 2\right]$, these bifurcations appear for values of $\lambda$ different to zero. For $\alpha$ close to the white noise $(\alpha=0.5)$ a bifurcationlike phenomena induced by noise is shown in Figs. 2 and 3. It consists of the separation of branches of the $f_{+} f_{-}$and $f_{-} f_{+}$maps that were unified by the noise. This kind of behavior is typical of systems with noise. Instead of bifurcations, some authors refers to it as multifurcation [9].

In the strict $\alpha=0$ limit, several tangent bifurcations are observed. They come from the superposition of the tangent bifurcations existing in the quartic maps $f_{+} f_{-}$ and $f_{-} f_{+}$. For $\alpha$ very small but different to zero these bifurcations disappear since the first transient from the attractors of $f_{+} f_{-}\left(f_{-} f_{+}\right)$to those of $f_{-} f_{+}\left(f_{+} f_{-}\right)$ coincides with the unstable state suppressed in the tangent bifurcation. This fact is illustrated in Figs. 4, 5, and 6. In Fig. 4, we show the tangent bifurcation appearing in the $\alpha \rightarrow 0$ limit. Figures 5 and 6 show the evolution 

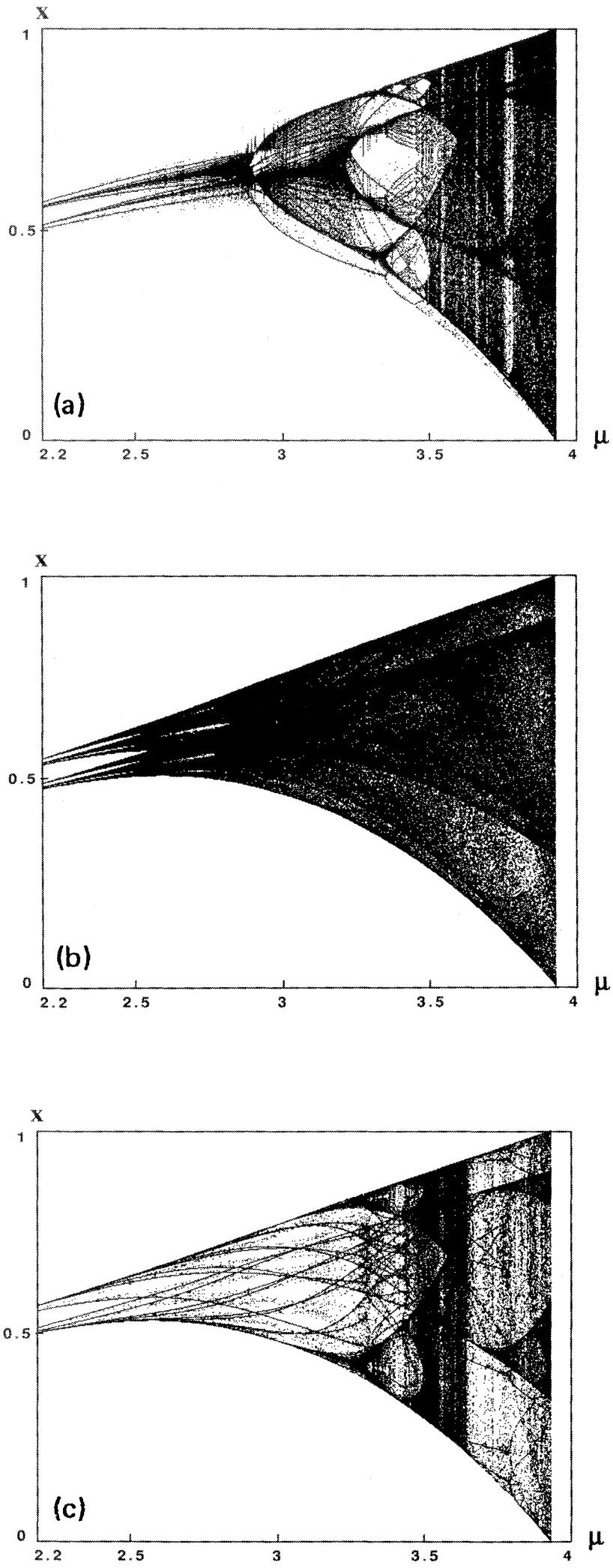

FIG. 1. Bifurcation diagrams of the noisy logistic map. Values of parameters are $\Delta=0.05$ and $\alpha=0.88$ (a), 0.5 (b), 0.01 (c).

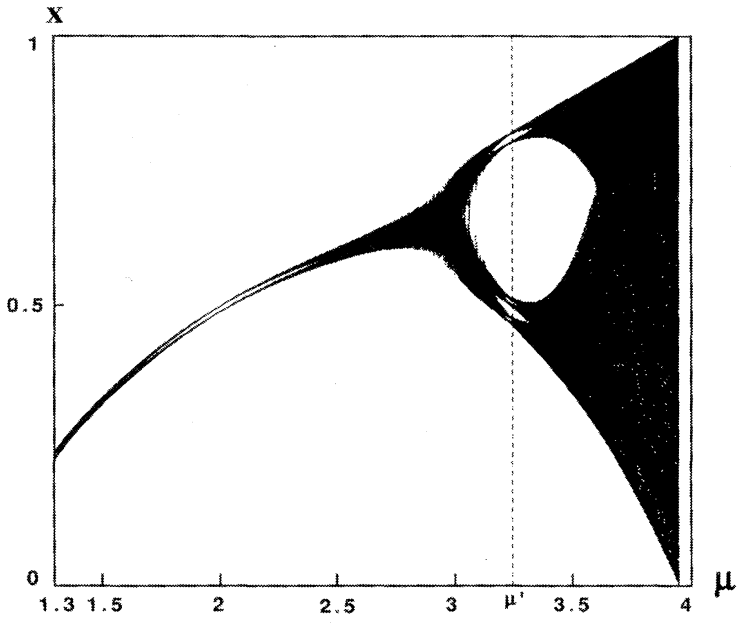

FIG. 2. Bifurcation diagram of the logistic map with dichotomic noise with $\Delta=0.01$ in the white-noise case $\alpha=\frac{1}{2}$. The dashed line corresponds to $\mu^{\prime}=3.25$. The invariant density for this value is shown in Fig. 3(d).

of the invariant density with $\alpha$ for two closed points, below and above the bifurcation point. We can observe that for $\alpha$ different to zero, the unstable attractor of the case $\alpha=0$ appears with small probability. Hence, bifurcation exists only in the strict limit $\alpha \rightarrow 0$. In this case the noise suppresses the bifurcation.

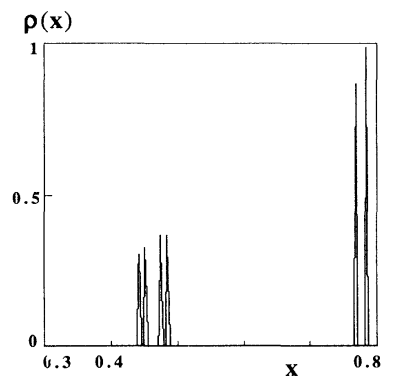

(d)

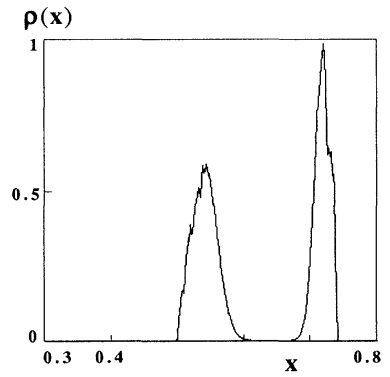

(a)

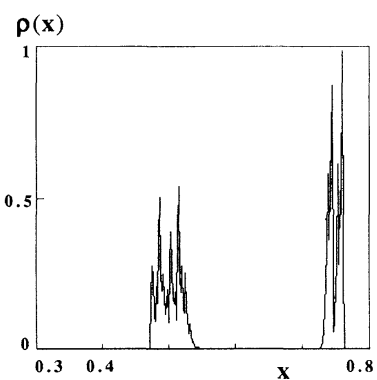

(c)

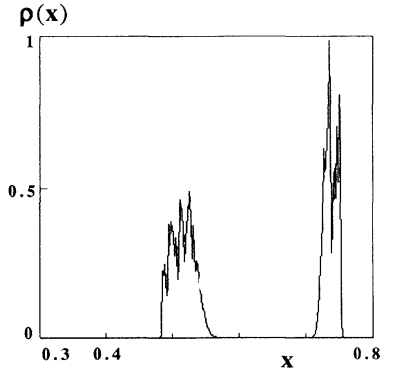

(b)
FIG. 3. Invariant densities for $\mu=3.08$ (a), 3.12 (b), 3.15 (c), and 3.25 (d) in the white-noise case $\left(\alpha=\frac{1}{2}\right)$ with intensity $\Delta=0.01$. All are below the point $\mu^{\prime}$ given in the Fig. 2 . 


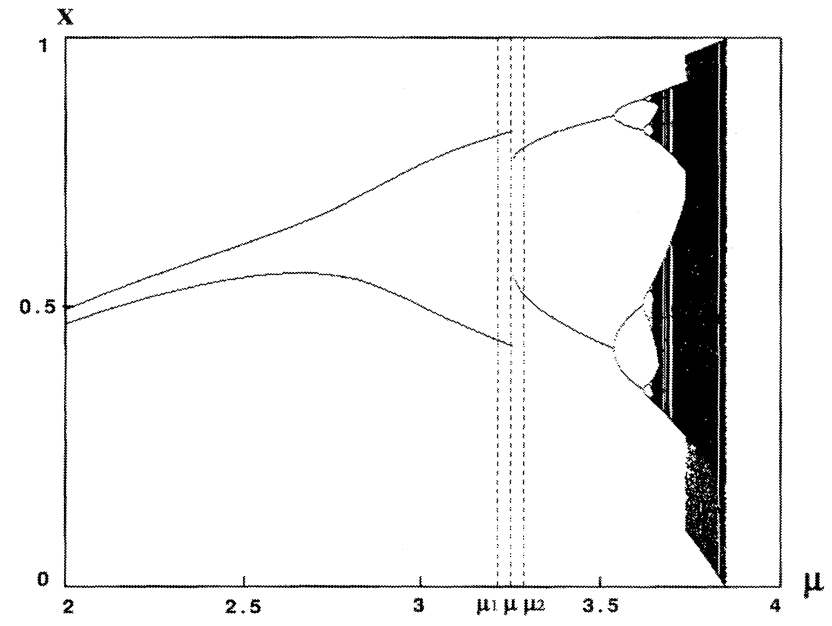

FIG. 4. Bifurcation diagram of the logistic map with dichotomic noise with $\Delta=0.025$ in the limit $\alpha \rightarrow 0$. A tangent bifurcation at $\mu=3.2736$ is shown. The initial condition is kept constant $\left(x_{0}=0.5\right)$ and the first value of the noise is $-\Delta$.

\section{STABILITY VERSUS CORRELATION TIME}

When analyzing the effect of noise on continuous nonlinear systems an interesting problem is to estimate changes in stability due to changes in the correlation time. Since in most of the studied cases the noise does not change the structure of the deterministic attractors a good measure of the stability changes is given by some

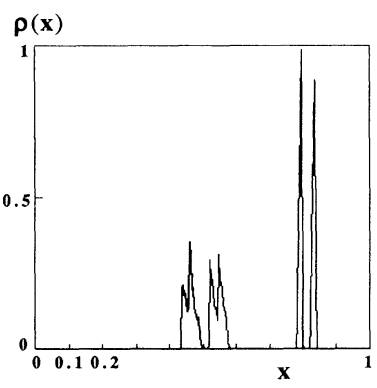

(d)

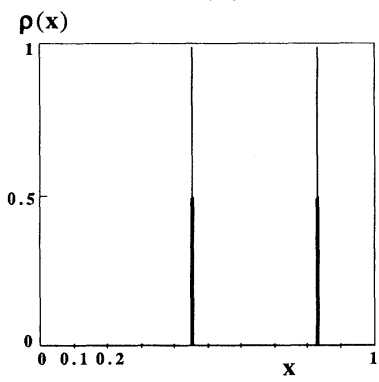

(a)

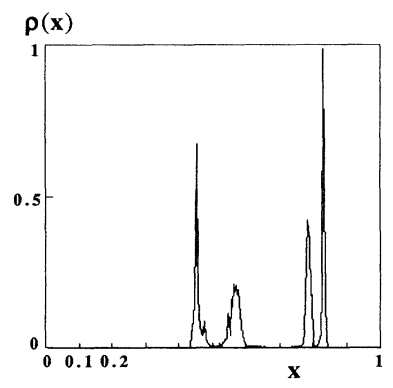

(c)

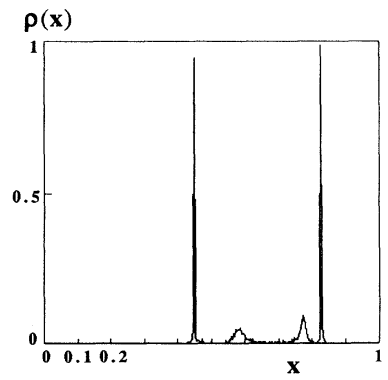

(b)
FIG. 5. Evolution of the invariant density with $\alpha$ [0 (a), 0.01 (b), 0.1 (c), 0.5 (d)] in a point $\mu_{1}=3.27$ below the tangent bifurcation shown in Fig. 4.

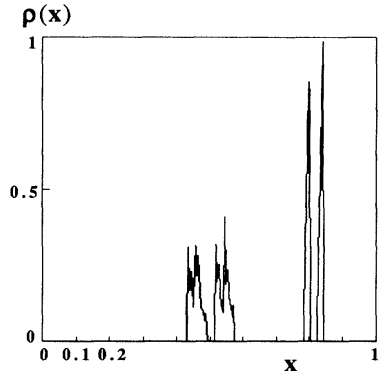

(d)

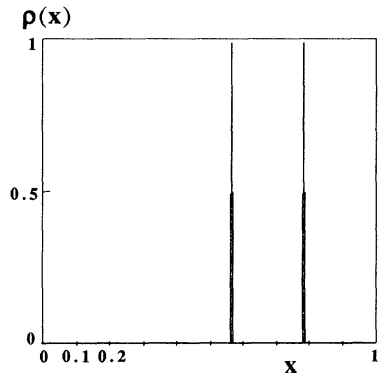

(a)

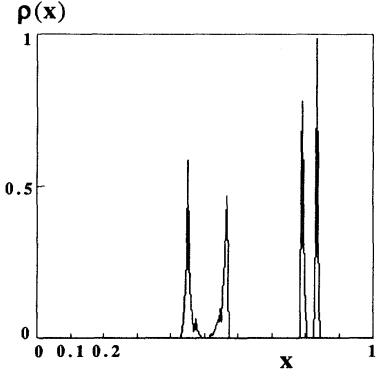

(c)

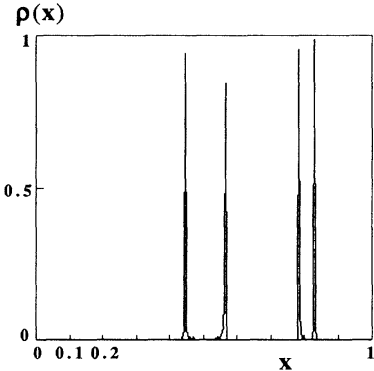

(b)
FIG. 6. Evolution of the invariant density with $\alpha$ [0 (a), 0.01 (b), 0.1 (c), 0.5 (d)] in a point $\mu_{2}=3.28$ above the tangent bifurcation shown in Fig. 4.

parameter measuring the intensity of fluctuations, such as, for example, variances or relative variances (variance to mean ratio, etc.).

In the present work, the structure of deterministic attractors are strongly modified and then estimators of the system stability must be used. Following the suggestions

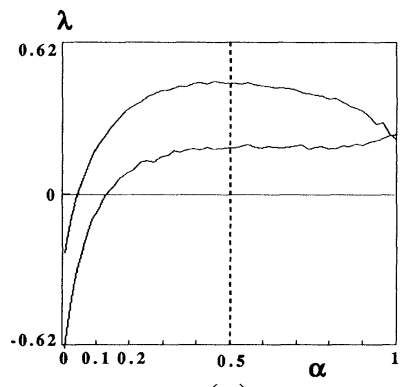

(a)

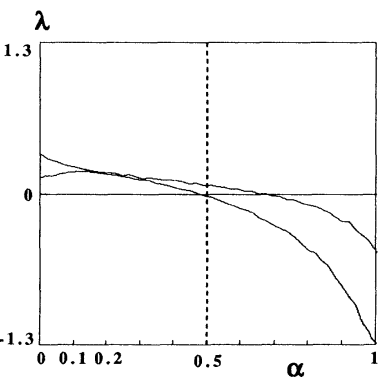

(b)

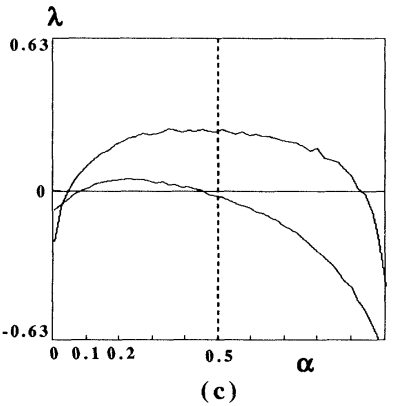

FIG. 7. Lyapunov exponent versus correlation time for the noisy logistic map with $\Delta=0.05$ in three situations corresponding to different values of $\mu[3.6,3.7$ (a); 3.41, 3.45 (b); and 3.35, $3.65(\mathrm{c})]$. 
of Ref. [8] we use Lyapunov exponents as such estimators. In deterministic systems Lyapunov exponents have a clear meaning as indicators of chaos. In noisy systems Lyapunov exponents give average stability properties of the orbits and then they are adequate for our purposes.

In order to illustrate the degree of instability induced by a colored noise let us consider as a first example the $\alpha \rightarrow 1$ limit studied in the last section. As we have said, the invariant measure and Lyapunov exponents in this case are given by an arithmetic mean of two logistic maps with control parameters shifted by $+\Delta$ and $-\Delta$. Since the structure of the attractors is very sensitive to changes in the control parameter, strong changes of the original attractors are possible even with low intensity. Moreover, these changes are in all possible directions. For instance, changes from chaotic to regular behavior, happen when the control parameter shifts from chaotic to regular motion.

In Fig. 7 we plot the Lyapunov exponent versus the correlation time in several situations. As we show, the behavior when changing the correlation time is not uniform. In Fig. 7(a) we go from a very stable configuration in the $\alpha \rightarrow 0$ limit to a chaotic situation in the $\alpha \rightarrow 1$ limit. The Lyapunov exponent always grows and as a consequence, the stability is monotically decreasing.

Figure $7(\mathrm{~b})$ is reciprocal and represents cases of increased stability with $\alpha$. Figure 7(c) corresponds to a case in which the stability in both deterministic limits is similar. Now there is a loss of stability when going from the above-mentioned limits to the white-noise case ( $\alpha=\frac{1}{2}$ ), but the point of maximum Lyapunov exponent does not always coincide with the exact white-noise situation, $\alpha=\frac{1}{2}$. In Fig. 8, we show values of $\alpha$ corresponding to a maximum in the Lyapunov exponent $\left(\alpha_{m}\right)$. Excluding the interval depicted in this figure, the maximum of the Lyapunov exponents are always located at $\alpha=1$. This fact is important in order to explain the behavior found in the white-noise case as we will see in Sec. V.

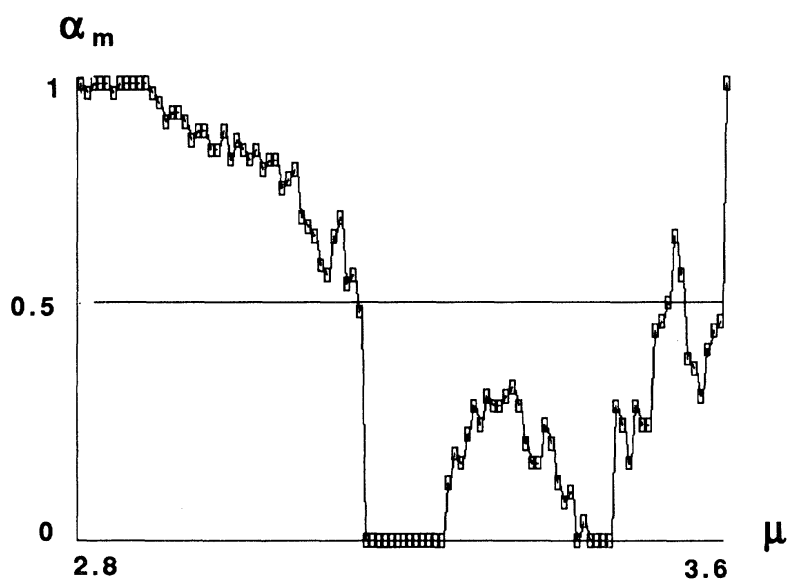

FIG. 8. Location of the maximum of the Lyapunov exponent $\alpha_{m}$ for the noisy logistic map $(\Delta=0.05)$ with $\mu$ varying in the interval $(2.8,3.6)$.
For the sake of comparison with the one-dimensional (1D) continuous model, we note that in such a model a colored noise always stabilizes the effect of a white noise [10]. The situation with 1D maps is, as we showed, very different. On the other hand, it is known that memory effects on maps may induce strong changes [4]. Hence, our results are expected since a colored noise introduced memory into the system.

\section{THE WHITE-NOISE CASE}

In the white-noise case, with $\alpha=\frac{1}{2}$, the equation for the invariant density takes a simple form:

$\rho(x)=\int\left\{\frac{1}{2} \delta\left(x-f_{+}(y)\right)+\frac{1}{2} \delta\left(x-f_{-}(y)\right)\right\} \rho(y) d y$,

but no analytical solutions are found. Possibly a perturbative analysis for small intensity $\Delta$ would give some approach in the $\Delta \rightarrow 0$ case but in general no analytical methods are available for solving the equation. However, we are able to identify and even to predict the peaks appearing in the invariant density (see Fig. 9). Since in a white noise all sequences are equally probable, one would expect a smooth shape of the invariant density. Nevertheless, one finds a rich variety of peaks, indicating some kind of resonance induced by noise. Plotting the invariant density for several values of $\alpha$ it is possible to observe the evolution of such peaks. In the limits $\alpha \rightarrow 0$ and $\alpha \rightarrow 1$ we have identified these in the Sec. II as attractors of single maps $f_{+}, f_{-}$or their compositions $f_{+} f_{-}$,

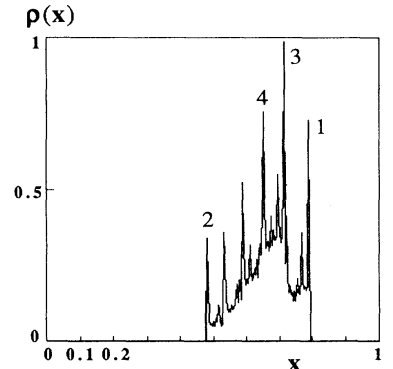

(d)

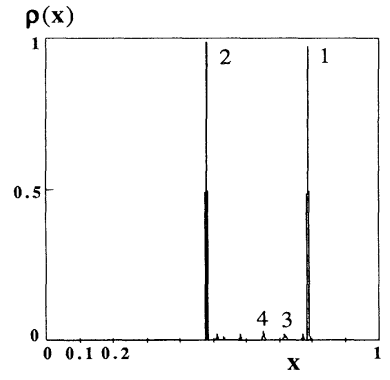

(a)

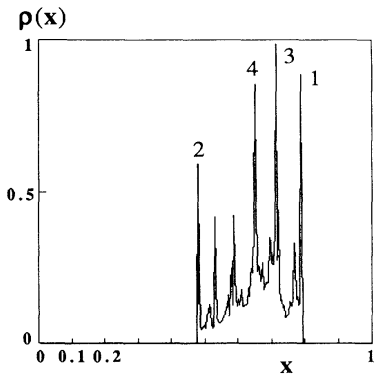

(c)

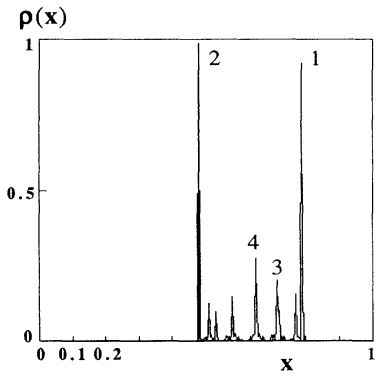

(b)
FIG. 9. Evolution of the invariant density of the noisy map with $\Delta=0.05$ through four values of $\alpha$ [0.01 (a), 0.1 (b), 0.4 (c), 0.5 (d)]. Peaks are identified with a number. 
$f_{-} f_{+}$. Also, we know that the small peaks are transients from one attractor to others. Going to the whitenoise case $\alpha \rightarrow \frac{1}{2}$ from the $\alpha \rightarrow 0$ limit it can be seen in Fig. 9 that some of the large peaks found in this limit disappear while some other small peaks grow. At the end, $\alpha=\frac{1}{2}$, we obtain a configuration of identified peaks with more or less intensity. This behavior with competence between attractors can be understood computing the Lyapunov exponent. We have analyzed this process in several cases concluding that the more stable attractor (attractor with the small Lyapunov exponent) always remains and, moreover, peaks associated with the transient from it grow. Hence, we conclude that, at least with the dichotomic noise, peaks appearing in the invariant density can be identified and analyzed. Repeating the same analysis, we have found the same results with a three-level noise. In this case, the analysis is much more complicated because the number of possible attractors increases but the conclusions are basically identical. A similar analysis with a general multilevel noise holds.

Hence, we conclude that the effect of a multilevel noise on discrete maps can be analyzed as superposition of competing attractors corresponding to maps found in the deterministic limits. Rather than a description based on shifts and broadening of bifurcations, this picture seems to be more precise.

\section{ACKNOWLEDGMENTS}

Financial support from Universidad de Cantabria and DGICYT (Spain) Project NO. PS90-0098 is acknowledged.
[1] S. J. Linz and M. Lucke, Phys. Rev. A 33, 2694 (1986).

[2] J. Crutchfield, M. Nauenberg, and J. Rudnick, Phys. Rev. Lett. 46, 933 (1981); B. Shraiman, C. W. Wayne, and P. C. Martin, ibid. 46, 935 (1981).

[3] P. Reimann and P. Talkner, Helv. Phys. Acta 64, 946 (1991); R. Graham, A. Hamm, and T. Tel, Phys. Rev. Lett. 66, 3089 (1991).

[4] E. Fick, M. Fick, and G. Hausmann, Phys. Rev. A 44, 2469 (1991).

[5] A. J. Irwin, S. J. Fraser, and R. Kapral, Phys. Rev. Lett. 64, 2343 (1990); S. J. Fraser and R. Kapral, Phys. Rev. A 45, 3412 (1992).

[6] C. Van den Broeck and P. Hanggi, Phys. Rev. A 30, 2730
(1984); J. Masoliver, K. Lindenberg, and B. West, ibid. 33, 2177 (1986); M. A. Rodriguez and L. Pesquera, ibid. 34, 4532 (1986).

[7] J. M. Gutiérrez, A. Iglesias, and M. A. Rodriguez, in The 2nd Granada Lectures in Computational Physics (World Scientific, Singapore, in press).

[8] L. Schimansky-Geier and H. Herzel (unpublished).

[9] T. Kapitaniak, Chaos in Systems with Noise (World Scientific, Singapore, 1990), and references included therein.

[10] J. M. Sancho, Stochastic Processes Applied to Physics (World Scientific, Singapore, 1984), p. 96. 